\title{
Beam quality improvement in the later stage of radiation pressure acceleration
}

\author{
Peng Liu, ${ }^{1}$ Junfan Qu, ${ }^{1}$ Xiangyang Liu, ${ }^{1,2}$ Xiaofeng $\mathrm{Li},{ }^{3,4}$ \\ Ling Cai, ${ }^{1}$ Jingyi Tang, ${ }^{1}$ and Qing Kong ${ }^{1, *}$ \\ ${ }^{1}$ Key Laboratory of Nuclear Physics and Ion-beam Application (MOE), Institute of Modern Physics, \\ Department of Nuclear Science and Technology, Fudan University, Shanghai 200433, China \\ ${ }^{2}$ Key Laboratory of Pulsed Power, Institute of Fluid Physics, CAEP, Mianyang 621900, China \\ ${ }^{3}$ Collaborative Innovation Center of IFSA (CICIFSA), Shanghai Jiao Tong University, \\ Shanghai 200240, China \\ ${ }^{4}$ Key Laboratory for Laser Plasmas (MOE), School of Physics and Astronomy, \\ Shanghai Jiao Tong University, Shanghai 200240, China
}

(Received 23 September 2019; published 15 January 2020)

\begin{abstract}
It is known that high quality proton beams can be produced in the radiation pressure acceleration (RPA) by using a circularly polarized ultraintense super-Gaussian laser. However, a transverse mismatching phenomenon between the laser intensity profile and the particle spatial distribution appears in the later stage of RPA, which leads to a decompression of proton beam and broadening of the energy spectrum. To weaken this effect, a new scheme with an additional plasma channel located behind a thin hydrogen foil is proposed. It is found that a good local matching can be maintained when the laser pulse propagates in the channel, which contributes to a stable RPA for a longer time. Two-dimensional particle-in-cell simulations show that the proton beam has a peak energy of $2.0 \mathrm{GeV}$ and energy spread of $13.8 \%$ at $t=300 \mathrm{fs}$. With further acceleration until $t=500 \mathrm{fs}$, a better quality beam with about $40 \%$ increase in peak energy and $26.2 \%$ improvement in energy conversion efficiency for high-energy protons $(\geq 1.5 \mathrm{GeV})$ can be obtained finally. Meanwhile, the energy spread drops from $100 \%$ to $28.5 \%$. This work may provide a more promising way to generate the high quality proton beam.
\end{abstract}

DOI: 10.1103/PhysRevAccelBeams.23.011303

\section{INTRODUCTION}

Laser-driven ion acceleration has attracted a great deal of attention because of its extensive applications and micro acceleration size. The high quality ion beams are useful in studying new ignition schemes of inertia confinement fusion (ICF) [1-3], cancer therapy [4], nuclear physics [5], and proton imaging [6]. Several implemented and proposed schemes for ion acceleration have been widely investigated theoretically and experimentally, including target normal sheath acceleration (TNSA) [7-9], radiation pressure acceleration (RPA) [10-17], breakout afterburner acceleration (BOA) [18-20], Coulomb explosion (CE) [21,22], and collisionless shock acceleration $[23,24]$. Up to now, the maximum proton energy of $94 \mathrm{MeV}$ and carbon ions energy of $48 \mathrm{MeV} / \mathrm{u}$ have been achieved experimentally via a

\footnotetext{
*Corresponding author. qkong@fudan.edu.cn

Published by the American Physical Society under the terms of the Creative Commons Attribution 4.0 International license. Further distribution of this work must maintain attribution to the author(s) and the published article's title, journal citation, and DOI.
}

hybrid RPA-TNSA scheme $[25,26]$. However, the ion beams generated by these schemes do not always meet the needs of practical applications. As far as we know, some numerical studies aimed at designing target structure have improved the beam quality of TNAS or BOA protons, for example, using a holed target or a plasma channel [27-30].

Compared with other schemes, the RPA can produce more monoenergetic and collimated proton beams, and it is also considered as one of the most promising schemes so far. As pointed out in recent publications [11-17], a circularly polarized (CP) laser pulse with the electrical field $E_{L}=E_{0}[\sin (\omega t) \hat{y}+\cos (\omega t) \hat{z}]$ is usually used in the RPA, and its ponderomotive force can be expressed as [11]

$$
f_{p}=-\frac{e^{2}}{4 m_{e} \omega^{2}} \nabla E_{0}^{2}
$$

where $m_{e}, \omega$, and $-e$ are the electron rest mass, laser frequency, and electron charge, respectively; $E_{0}$ is the laser electric field amplitude. Because of the absence of the oscillating component in Eq. (1), the longitudinal ponderomotive force can directly push the electron layer forward and form the charge separation field, which is strong enough to drive the protons to move together and they 
constitute a quasineutral plasma slab. However, the ultrathin foil employed in the RPA scheme is very susceptible to the transverse instabilities [31], which leads to deterioration of beam quality, especially in the later stage of acceleration [16]. Several schemes using either a shaped foil target [32] or a tailored pinhole target [33] to enhance the monochromaticity and collimation have been investigated numerically. Recently, it was reported that proton acceleration driven by laser pulses with a super-Gaussian profile can suppress target deformation in the RPA scheme [34], and Qiao et al. have attained about $1.35 \mathrm{GeV}$ proton beams by a transverse fourth-order super-Gaussian laser at $t=107 \mathrm{fs}$ [14]. To our best knowledge, most studies about the RPA scheme have followed the acceleration for a limited time duration; there are a few simulations that have focused on laser intensity evolution and ion beam quality in the later stage. By our simulations, there is still more than $30 \%$ of the laser energy left when the laser interacts with foil for about 150 femtoseconds; it peaks our interest in further studying what will happen after protons have obtained energies in $\mathrm{GeV}$ magnitude.

In this paper, inspired by above work, we numerically investigate a typical RPA process in detail, and we find that even in the case of super-Gaussian laser driven proton acceleration, there is still a mismatching phenomenon in the later stage, i.e., where the electron density is high, the laser intensity is very weak. This leads to a rapid decompression of the proton beam and broadening of the energy spectrum. In order to weaken this mismatching effect and achieve efficient RPA, we suggest using an additional plasma channel, which is located behind a thin hydrogen foil. With the aid of two-dimensional (2D) particle-in-cell (PIC) simulations, it is found that a local matching phenomenon reappears when the laser pulse propagates in the channel, and a stable RPA can last for a longer time. As a consequence, the beam quality of accelerated protons is improved effectively.

\section{THE MISMATCHING EFFECT IN RPA}

We carry out a typical 2D PIC simulation to show a general picture of the RPA scheme by relativistic electromagnetic code EPOCH [35]. A normalized amplitude of $\mathrm{CP}$ laser pulse at the focus is $a=a_{0} \exp \left(-r^{4} / \sigma_{L}^{4}\right)$, where $a_{0}=e E_{0} / m_{e} \omega c=100$ with $c$ being light speed in vacuum, $\sigma_{L}=8 \lambda$ is the waist radius and $\lambda=1 \mu \mathrm{m}$ is the laser wavelength, and the corresponding laser intensity is $I=2.74 \times 10^{22} \mathrm{~W} / \mathrm{cm}^{2}$. The laser pulse has a trapezoidal temporal intensity profile and the pulse duration is $\tau=$ $12 T_{0}$ where rising time and falling time are both $1 T_{0}$, where $T_{0}=\lambda / c$ is laser period. The hydrogen foil target is initially located in the region $5.6 \lambda \leq x \leq 6.0 \lambda$ with the density of $n_{e}=75 n_{c r}$, where $n_{c r}=\varepsilon_{0} m_{e} \omega^{2} / e^{2}$ is the critical density. That is, the thickness of the hydrogen foil target is $l_{0}=0.4 \lambda$, which satisfies the optimum target thickness relation $D=\left(a_{0} / \pi\right)\left(n_{c r} / n_{e}\right) \lambda$ [11], and the width of the foil is $30 \lambda$ (i.e., $-15 \lambda \leq y \leq 15 \lambda$ ). The total simulation box is $90 \lambda(x) \times 30 \lambda(y)$, which corresponds to a $9000 \times 3000$ cells moving window, and 200 macroparticles are included in every cell. The laser pulse enters the simulation box from the left boundary at $t=0$. The open and the absorbing boundary conditions are adopted for the simulation particles and the electromagnetic wave, respectively.

When a CP laser pulse impinges on the foil target, the protons undergo a stable RPA process in the early stage, and a clear quasimonoenergetic peak of protons can be seen at $t=80 \mathrm{fs}$, as presented in Fig. 1. At this time, the peak energy is about $0.75 \mathrm{GeV}$ and the full-width-at-halfmaximum (FWHM) energy spread is $\Delta E / E \approx 16.2 \%$. Ideally, the proton beam can continuously obtain energy from the laser pulse until the laser energy is depleted finally, and there should always be a quasimonoenergetic peak during the acceleration. However, a broad proton energy spectrum is observed at $t=150 \mathrm{fs}$. Although the peak energy is up to about $1.21 \mathrm{GeV}$ for this time, the energy spread increases to about $19.8 \%$. We can hardly see a quasimonoenergetic peak in Fig. 1 at the time $t=300 \mathrm{fs}$, and final energy spread of the proton beam is more than $60 \%$. It is clear that the stable RPA is almost over in the later stage.

In order to reveal this physical process during the later stage of RPA, we present the snapshots of electron density, proton density, and laser intensity in Fig. 2 at different moments. We see that the compressed proton and electron layers detach from the foil target at $t=80 \mathrm{fs}$, which is consistent with the results of Ref. [14]. Both the proton and electron layers are very thin and flat. At the same time, the profile of laser intensity $\left(I_{L}=E_{y}^{2}+E_{z}^{2}\right)$ matches with the distribution of electron density well, and this local matching makes proton acceleration very stable now. The transverse distribution of electron density keeps evolving during

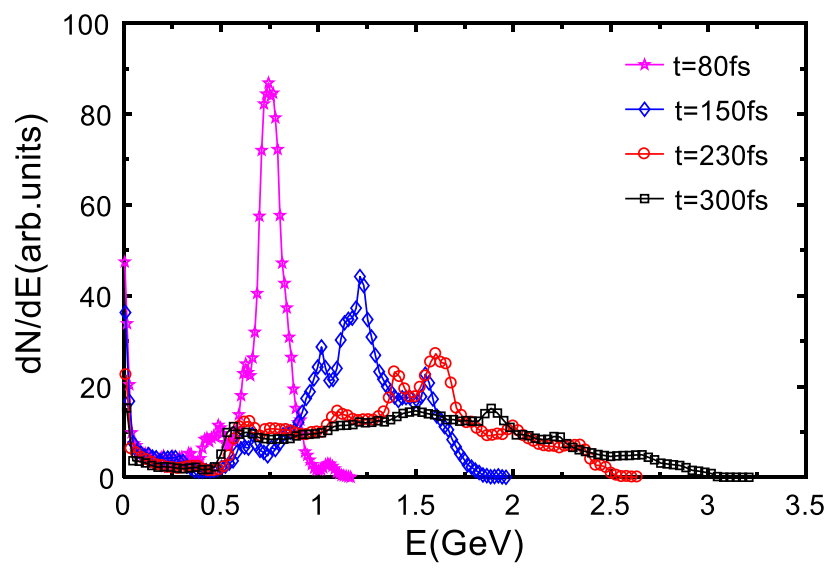

FIG. 1. Energy spectrum of protons for a common RPA at four time instants: $t=80 \mathrm{fs}, t=150 \mathrm{fs}, t=230 \mathrm{fs}$, and $t=300 \mathrm{fs}$. Here, the protons in the region of $-6 \lambda \leq y \leq 6 \lambda$ are chosen as statistical objects. 

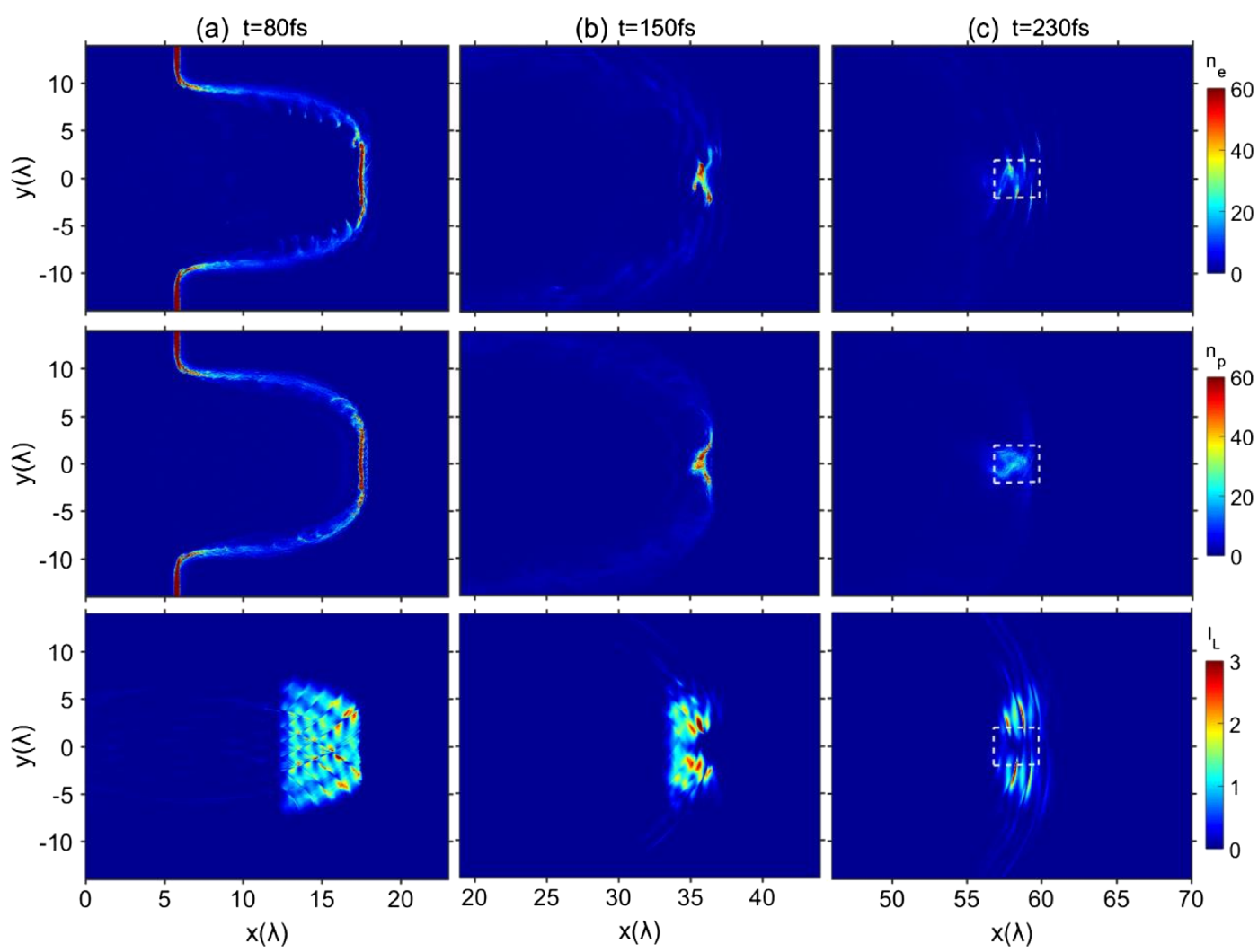

FIG. 2. Snapshots of electron density (normalized by $n_{c r}$ ), proton density (normalized by $n_{c r}$ ), and laser intensity (normalized by $E_{0}^{2}$ ) for a common RPA at three moments: $t=80 \mathrm{fs}(\mathrm{a}), t=150 \mathrm{fs}(\mathrm{b})$, and $t=230 \mathrm{fs}$ (c). The dashed line boxes indicate the position of particle beams.

the acceleration. At $t=150 \mathrm{fs}$, it is clear that the transverse size of the electron beam is reduced due to transverse instabilities [31], and the density at electron beam wings becomes lower. It is well known that the laser energy always tends to flow from the region of the higher plasma density to that of the lower one; that is, from the near-axis to the offaxis region in this work. It results in a hollowlike profile of laser intensity, as seen in Fig. 2(b); the evolution of the laser will further enhance the transverse diffusion of electrons at both wings, while the electrons near the central axis are compressed transversely. This is a self-organized process between the laser pulse and the electron beam. Meanwhile, we also see the evolution of proton density is similar to that of electron density. As a result, a mismatching phenomenon between the laser intensity and the particle beams appears. It should be noted that the mismatching effect comes from the laser energy flow, which in turn strengthens this effect. To indicate the degree of transverse mismatching clearly, we define a mismatching factor:

$$
\xi=\frac{\left|y_{L}-y_{e}\right|}{r_{e}},
$$

where $y_{L}$ and $y_{e}$ are the transverse positions of laser peak and electron beam center, respectively, and $r_{e}$ is the radius of the electron beam. The mismatching phenomenon has appeared at $t=150 \mathrm{fs}$, and we can get $\xi \approx 0.85$ from Eq. (2). Furthermore, this phenomenon becomes more and more obvious in the process of acceleration, and the laser pulse is divided into two beams located at both ends of the particle beams, as marked by dashed line boxes in Fig. 2. As we can see, the electron beam also evolves into several micro bunches and oscillates periodically at $t=230$ fs [see Fig. 2(c)], and the corresponding $\xi$ is about 1.28 for this moment. Once this occurs, the proton beam decompresses longitudinally, and the final energy spectrum is inevitably broadened, as shown in Fig. 1. As a conclusion, the broadening of the energy spectrum is due to the absence of light pressure, which is attributed to the mismatching effect. It should be remarked that the protons do not experience a $\mathrm{CE}$ $[21,22]$ in this case, because plasma slab is still quasineutral in the later period.

During this period, there is still $55 \%$ of the laser energy left at $t=80$ fs [see Fig. 3(a)], i.e., $\rho=E_{r} / E_{\text {init }}=55 \%$, where $E_{r}$ is the residual energy of the laser pulse and $E_{\text {init }}$ is the initial energy of the laser pulse. $\rho$ are about $32 \%$ and $20 \%$ at $t=150$ fs and $300 \mathrm{fs}$, respectively. PIC simulations show that the laser energy decreases slowly after about $t=200 \mathrm{fs}$, which implies that the interaction between the 


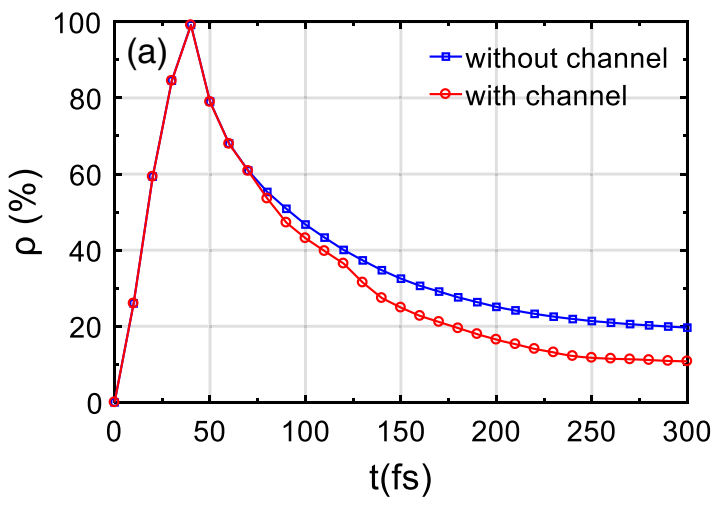

(b)
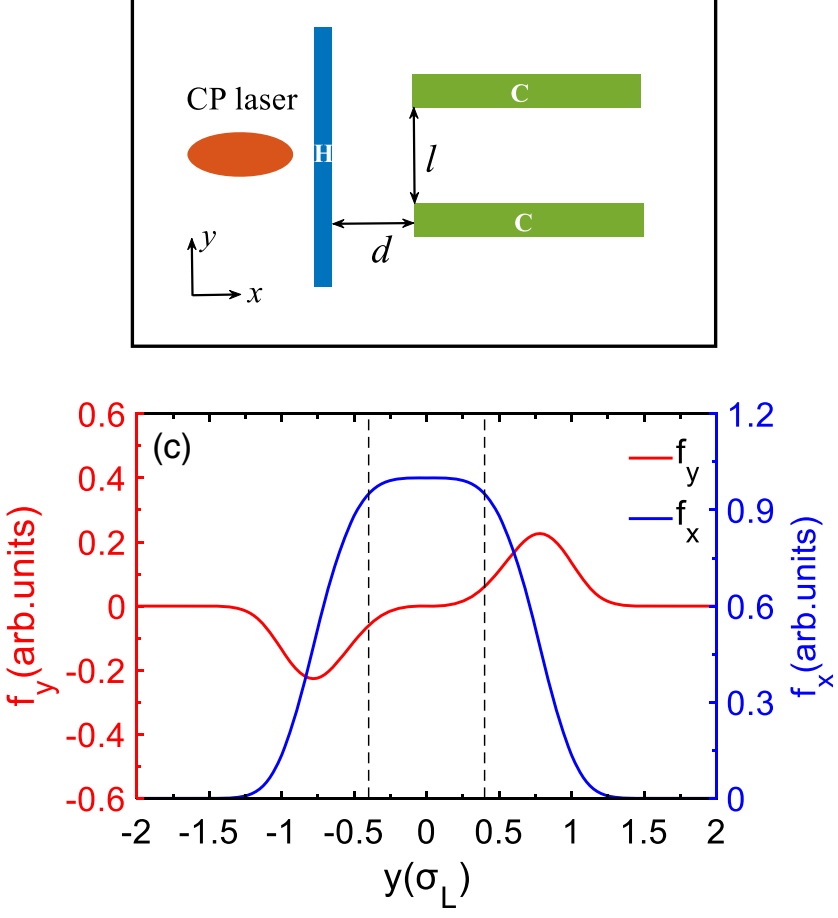

FIG. 3. (a) Temporal evolution of $\rho=E_{r} / E_{\text {init }}$, where $E_{r}$ is the residual energy of the laser pulse and $E_{\text {init }}$ is the initial energy of the laser pulse. (b) Schematic diagram of proton acceleration by the employment of an additional plasma channel. (c) Transverse and longitudinal ponderomotive force profile of a fourth-order super-Gaussian laser along the $y$ axis.

laser pulse and the particle beams becomes very weak and the stable RPA is close to the end.

\section{BEAM QUALITY IMPROVEMENT WITH CHANNEL}

Considering that the mismatching phenomenon is due to laser off-axis divergence in the above case, shown in Fig. 2, it is necessary to modulate the laser pulse to the desired shape and achieve the purpose of transverse matching. Here, we suggest using an additional plasma channel which is located behind the thin foil target, as shown in Fig. 3(b).
The distance between the channel and foil target and the channel width are denoted as $d$ and $l$, respectively. For a $\mathrm{CP}$ laser pulse with transverse fourth-order super-Gaussian intensity profile, its corresponding transverse and longitudinal ponderomotive force, according to Eq. (1), can be estimated as $f_{r} \sim r^{3} \exp \left(-2 r^{4} / \sigma_{L}^{4}\right)$ and $f_{x} \sim \exp \left(-2 r^{4} / \sigma_{L}^{4}\right)$, respectively. Figure 3(c) shows the distribution of this super-Gaussian laser pulse on the $x-y$ plane where $|y|=r$. It can be found that the transverse ponderomotive force first increases as $0 \leq r \leq \sqrt[4]{3 / 8} \sigma_{L}$, then decreases as $r \geq \sqrt[4]{3 / 8} \sigma_{L}$. Hence, the electrons close to $\sqrt[4]{3 / 8} \sigma_{L}$ are easily pushed away, while the electrons near the light axis feel much smaller transverse ponderomotive force; we also see that the transverse distribution of the longitudinal ponderomotive force shows a platformlike profile, which is relatively uniform between $-0.4 \sigma_{L}$ and $0.4 \sigma_{L}$, as presented by two black dashed lines in Fig. 3(c).

Based on the distribution characteristic of the ponderomotive force, a stable plasma slab with a width of about $0.8 \sigma_{L}$ may detach from the target during the early stage of acceleration, which is consistent with the PIC simulation results of Fig. 2(a) at $t=80$ fs. Thus, the width of the plasma channel should be larger than $0.8 \sigma_{L}$ to ensure that the plasma slab can enter the channel completely. Combined with the results of Fig. 1, the protons have experienced a stable RPA by the time $t=80 \mathrm{fs}$, and in order to well confine the laser and attain a high quality proton beam in the later stage of acceleration, the plasma channel should be near the particle beams' position of this time [see Fig. 2(a)]. Here, we choose $l=10 \lambda$ and $d=8 \lambda$ in the next simulation case with a channel.

In order to verify the effect of the plasma channel on proton acceleration, we have performed a series of $2 \mathrm{D}$ PIC simulations. In this scheme we proposed, the plasma channel which consists of fully ionized carbon ions and electrons is located at $14 \lambda \leq x \leq 50 \lambda$ with thickness $1.5 \lambda$. The initial density of carbon ion is chosen to be $60 n_{c r}$. For a clear comparison, the other parameters are the same as those in the case shown in Figs. 1 and 2.

Like the case without a channel, a typical RPA takes place outside the channel initially, then the laser and particle beams enter into the channel at about $t=70 \mathrm{fs}$, and they leave the channel at around $t=200 \mathrm{fs}$. Figure 4 shows the temporal evolution of electron density, proton density, and laser intensity. At $t=150 \mathrm{fs}$, the compressed electron and proton layers can be observed in Figs. 4(a) and 4(c). This quasineutral plasma slab is accelerated as a whole and remains highly overdense while its shape closely resembles the transverse laser profile [see Fig. 4(e)]. The laser profile in this scheme is very different from that in the case without a channel [Fig. 2(b)]. The laser divergence mentioned above is effectively weakened by the use of plasma channel, and we can attain $\xi \approx 0.05$ from Eq. (2) at this moment. Thus, the electron layer near the light axis can be continuously compressed and accelerated by the laser 

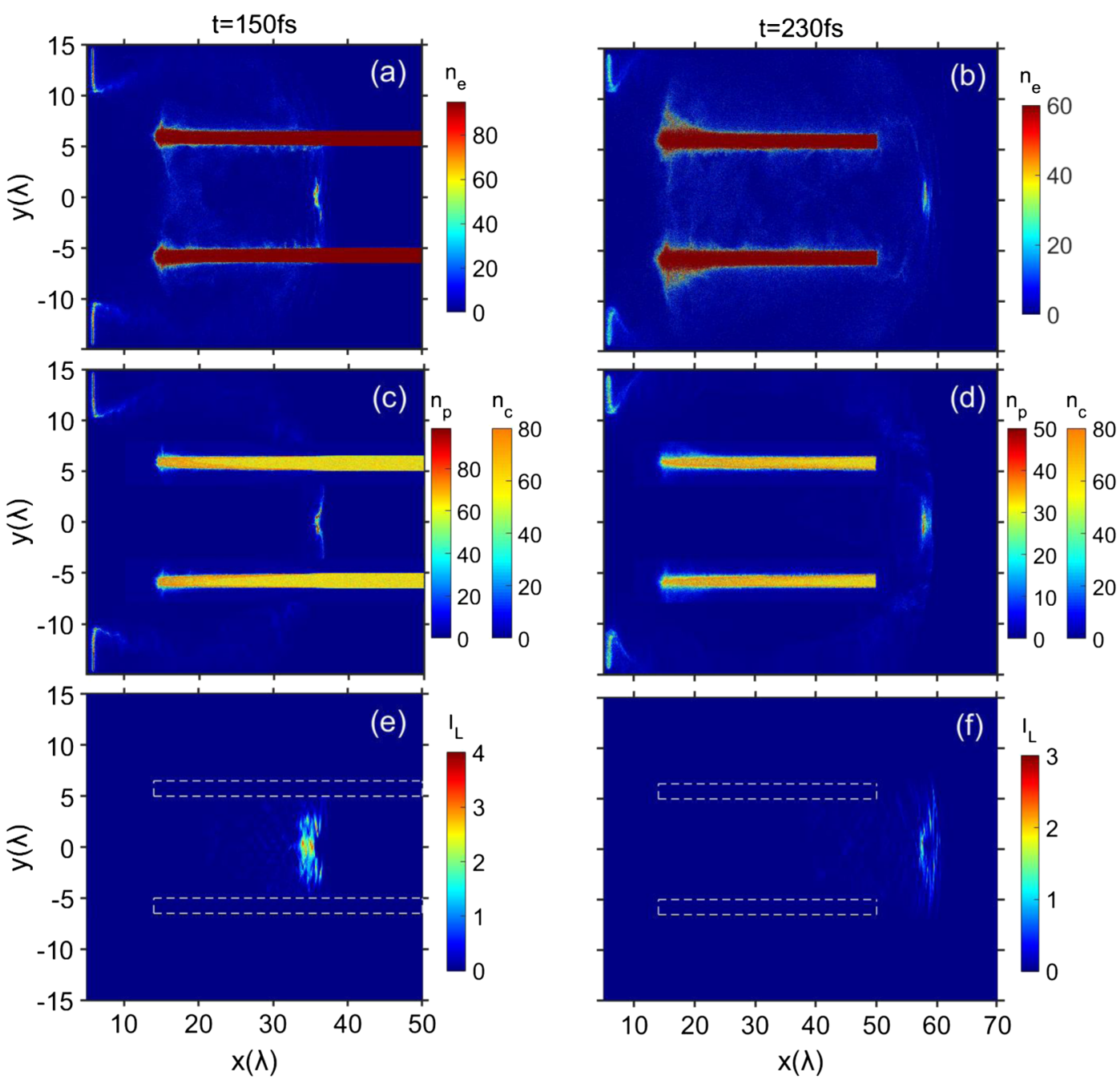

FIG. 4. Spatial distributions of electron density (a),(b), proton and carbon density (c),(d), and laser intensity (e),(f) for the case with a channel at two moments: $t=150 \mathrm{fs}$ and $t=230 \mathrm{fs}$. The color bars represent the particle density normalized by $n_{c r}$ and the laser intensity normalized by $E_{0}^{2}$. The dashed lines indicate the position of channel.

ponderomotive force, which favors the stable acceleration of protons. Compared with the case without a channel, both the electron and proton beams are still compact longitudinally when they travel in vacuum $(t=230 \mathrm{fs})$, as shown in Figs. 4(b) and 4(d), and the mismatching factor $\xi$ is about 0.08 for this time. Therefore, the good local matching in the case with a channel can support a stable RPA in the later stage. In this scheme we proposed, only less than $10 \%$ of the initial laser energy is left at the moment $t=300 \mathrm{fs}$ [see Fig. 3(a)], meaning a high energy conversion efficiency from laser pulse to particles. In short, the presence of a plasma channel can weaken this mismatching effect strongly and a more efficient RPA can be maintained for a longer time.

Figure 5 presents the further evolution of electron beam and laser intensity at $t=500 \mathrm{fs}$. We see that laser transverse divergence is very obvious in both cases, as shown in Fig. 5(a). The laser intensity in the case without a channel is higher than that in the case with a channel, which is consistent with the results of Fig. 3(a). This is because more laser energy is transferred to the particle beams in the channel. Meanwhile, the laser pulse center $(x \approx 140 \lambda)$ almost longitudinally surpasses the electron beams, which are located at about $117 \lambda \leq x \leq 138 \lambda$ for both cases [see Fig. 5(b)]. This implies that the interaction of the laser and the particle beams is very weak after $t=300 \mathrm{fs}$, and the absence of light pressure leads to the expansion of electron beams.

As expected, the protons from the case with a channel gain more uniform energies because of a much more stable and synchronous acceleration, and the very clear quasimonoenergetic peaks can be seen at different moments from Fig. 6(a). At $t=150$ fs, the FWHM energy spread drops to $10.8 \%$. After the proton beam leaves the channel (such as $t=230 \mathrm{fs}$ ), the peak energy of the proton beam is up to $1.75 \mathrm{GeV}$ with an energy spread of $\Delta E / E \approx 12.1 \%$. 

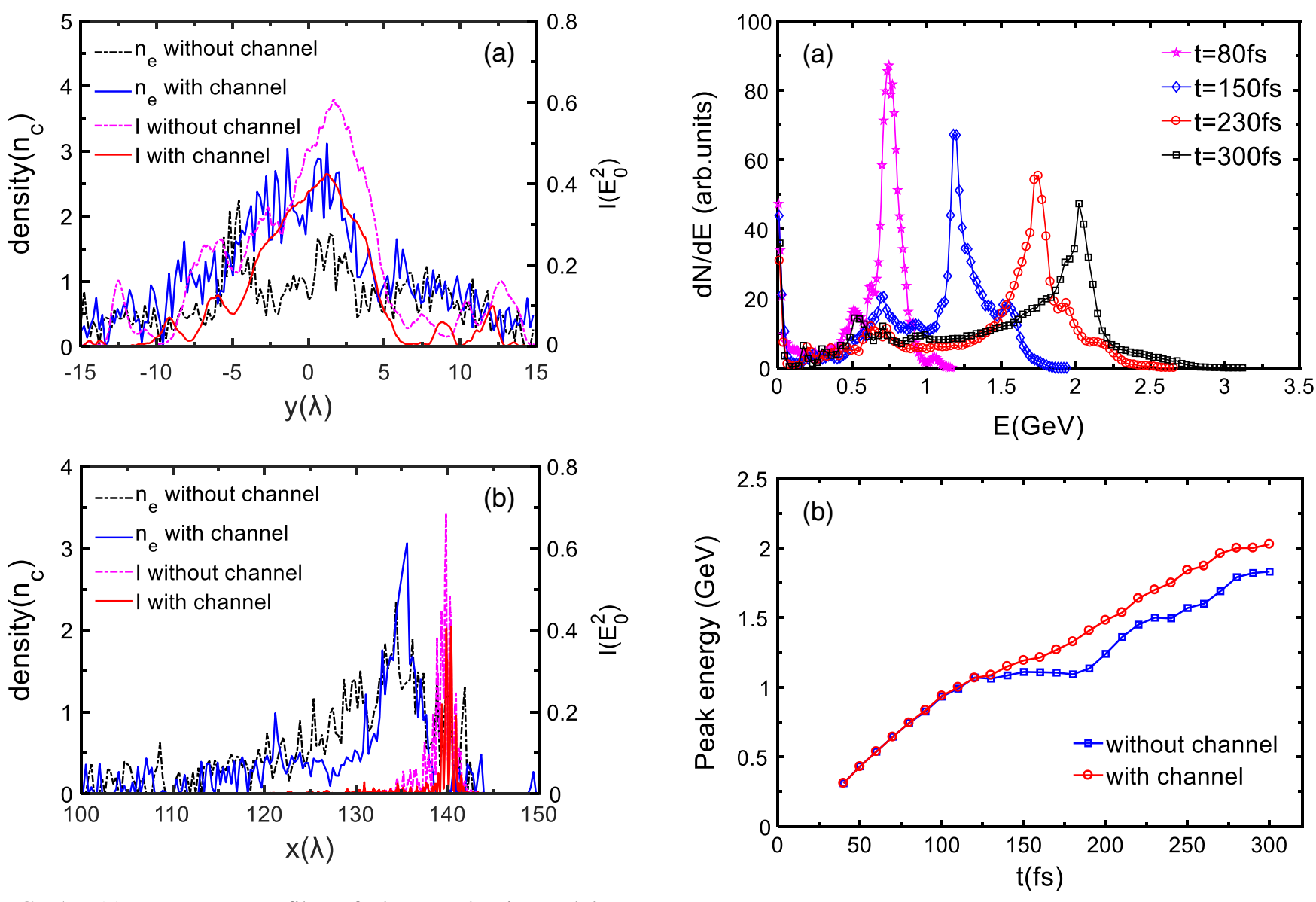

FIG. 5. (a) Transverse profiles of electron density and laser intensity along the $x=135 \lambda$ and $x=140 \lambda$, respectively. (b) Longitudinal profiles of electron density and laser intensity both along the $y=0$. Dashed and solid lines represent the cases without a channel and with a channel, respectively. Snapshots correspond to the moment $t=500 \mathrm{fs}$.

The peak of the energy spectrum is still very clear at $t=300 \mathrm{fs}$, and the peak energy reaches $2.0 \mathrm{GeV}$ though the energy spread slightly increases to about $13.8 \%$. By contrast, the energy spread in the case without a channel is about $25 \%$ at $t=230 \mathrm{fs}$, and more than $60 \%$ at the time $t=300 \mathrm{fs}$. Here, we choose the protons in the region of $-6 \lambda \leq y \leq 6 \lambda$ as the statistical objects. It is noteworthy that the energy spread of the energetic proton beams in this statistical region is almost the same as that in the whole simulation box.

Figure 6(b) shows the temporal evolution of the proton peak energy in both cases. Because of the mismatching effect in the case without a channel, only a small portion of protons is accelerated to high energy after $120 \mathrm{fs}$. Thus, the peak energy almost unchanged, keeping nearly constant from 120 to $180 \mathrm{fs}$, but the maximum energy increases a little. It leads to a rapid increase of energy spread during this period, as seen in Fig. 1. For the scheme we proposed, however, the peak energy keeps rising steadily and the energy spread remains lower until $t=300 \mathrm{fs}$. The energy conversion efficiency $\eta=E_{p} / E_{\text {init }}$ from laser to

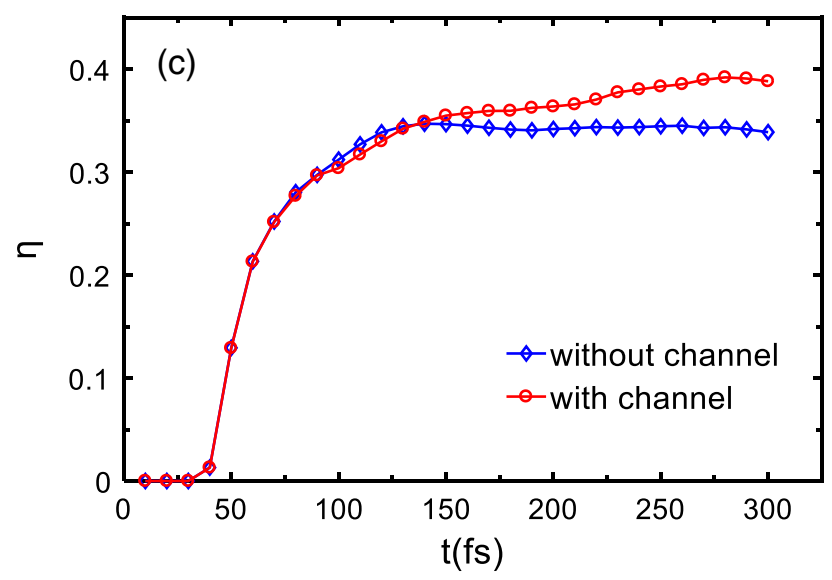

FIG. 6. (a) Energy spectrum of the proton for the case with a channel at four time instants: $t=80 \mathrm{fs}, t=150 \mathrm{fs}, t=230 \mathrm{fs}$, and $t=300 \mathrm{fs}$. Temporal evolution of peak energy (b) and energy conversion efficiency (c) for both cases, respectively. Here, the protons in the region of $-6 \lambda \leq y \leq 6 \lambda$ are chosen as statistical objects.

accelerated protons is shown in Fig. 6(c), where $E_{p}$ is the total kinetic energy of protons $(\geq 0.4 \mathrm{GeV})$. We see that there is no significant difference in $\eta$ for two cases during the early stage of acceleration. But in the later stage, especially after $t=150 \mathrm{fs}$, the $\eta$ in this scheme is higher 

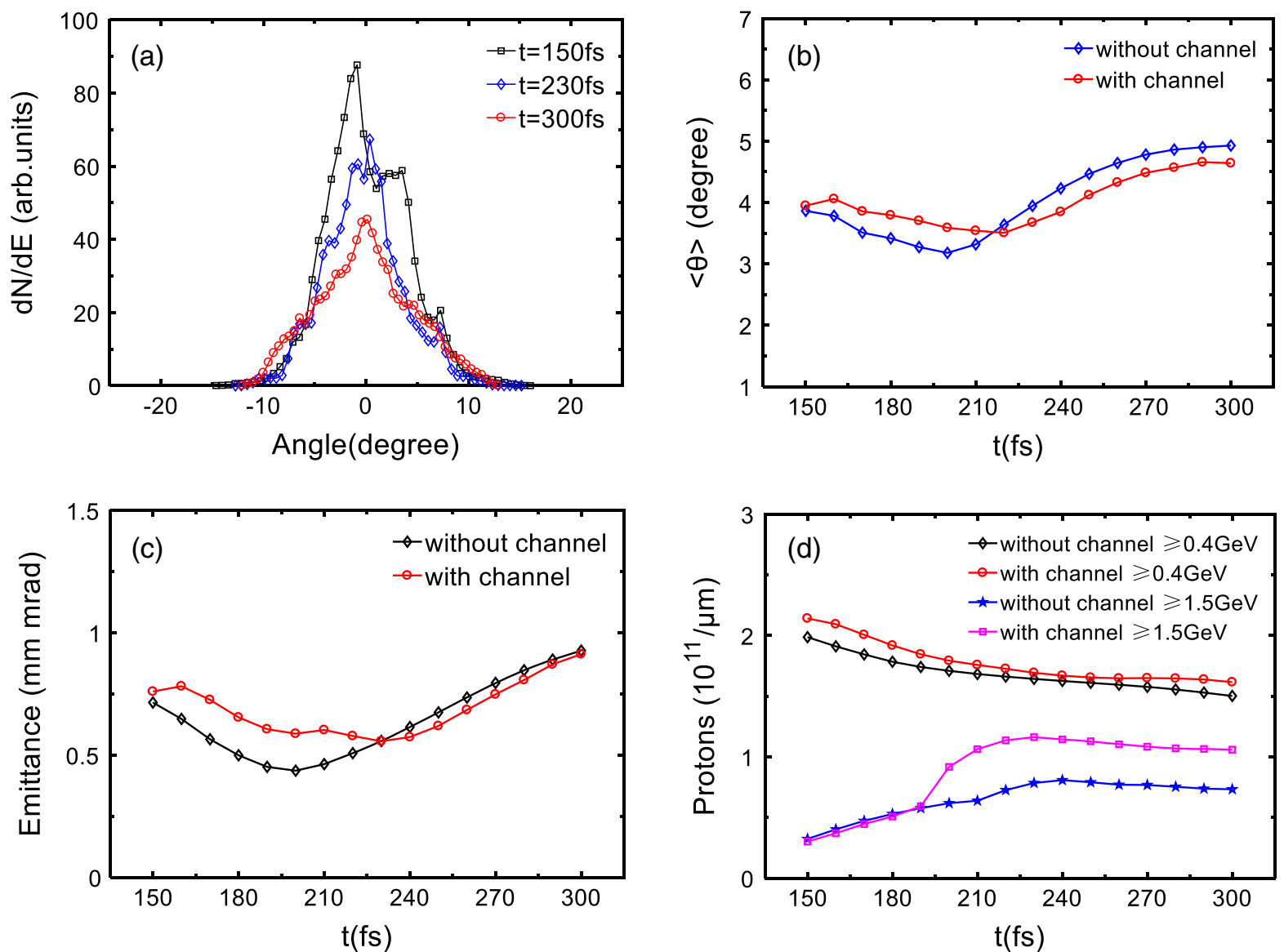

FIG. 7. (a) Angular spectrum of protons in the case with a channel at three moments: $t=150 \mathrm{fs}, t=230 \mathrm{fs}$, and $t=300 \mathrm{fs}$. Temporal evolution of average divergence angle (b) and transverse emittance (c) for the protons in both cases, respectively. (d) Number of protons for different energies (i.e., $\geq 0.4 \mathrm{GeV}$ and $\geq 1.5 \mathrm{GeV}$ ) in two cases. Here, we choose the protons in the region of $-6 \lambda \leq y \leq 6 \lambda$.

than that in the case without a channel, and it increases from $33.7 \%$ to $39.0 \%$ at $t=300$ fs.

Figure 7(a) presents the angular spectrum in the case with a channel, it is clear that most of the protons are concentrated between about $-8^{\circ}$ and $8^{\circ}$, and the peak values are all close to $0^{\circ}$, meaning that the proton beam is highly directed. The evolution of an average divergence angle $(\langle\theta\rangle)$ and the corresponding transverse emittance $(\varepsilon)$ of the protons in the later stage are shown in Figs. 7(b) and $7(\mathrm{c})$, respectively. We see that the collimation of the beam is improved by using the plasma channel. At $t=300 \mathrm{fs}$, the $\langle\theta\rangle$ and $\varepsilon$ are $4.5^{\circ}$ and $0.91 \mathrm{~mm} \mathrm{mrad}$, which are all lower than that in the case without a channel. Here, the $\langle\theta\rangle$ and $\varepsilon$ are calculated as follows [36]:

$$
\begin{gathered}
\langle\theta\rangle=\sqrt{\sum_{i=1}^{N}\left(\theta_{i}\right)^{2} / N}, \quad \theta_{i}=\tan ^{-1}\left(P_{y} / P_{x}\right), \\
\varepsilon=\frac{4}{N} \sqrt{\sum_{i=1}^{N}\left(y_{i}-\langle y\rangle\right)^{2}} \times \sqrt{\sum_{i=1}^{N}\left(\theta_{i}-\langle\theta\rangle\right)^{2}},
\end{gathered}
$$

where $N$ is the total number of the accelerated protons, and $P_{x}$ and $P_{y}$ are the momentum component in the $x$ and $y$ directions, respectively. $y_{i}$ represents the transverse positions of each proton, and $\langle y\rangle$ is the mean value of $y_{i}$. The number of protons drops in both cases because of the transverse expansion, as presented in Fig. 7(d). We see that the number of protons with energy greater than $0.4 \mathrm{GeV}$ increases slightly in this scheme. However, there is a significant improvement in the number of protons with much higher energies $(\geq 1.5 \mathrm{GeV})$, and the total number of these protons can be up to about $1.06 \times 10^{11} / \mu \mathrm{m}$ at $t=300 \mathrm{fs}$.

As we can see, the beam quality of protons changes slowly after $t=300$ fs according to Figs. 6 and 7. We also check the further evolution of the accelerated protons until the end of simulation ( $t=500 \mathrm{fs}$ ), and the final acceleration results of the two cases are given in Table I. It shows that there is a significant improvement in beam quality by the employment of a plasma channel. It should be remarked that the collimation of accelerated protons is improved slightly, but the final peak energy of the proton beam is about $40 \%$ higher than that in the case without a channel, and the energy spread drops from $100 \%$ to $28.5 \%$. 
TABLE I. The final peak energy $\left(E_{p}\right)$, energy spread $(\Delta E / E)$, average divergence angle $(\langle\theta\rangle)$, and transverse emittance $(\varepsilon)$ of all protons in both cases at $t=500 \mathrm{fs}$. The number $(N)$ and total energies $\left(E_{\text {tot }}\right)$ of high-energy protons with energy larger than $1.5 \mathrm{GeV}$ are also calculated.

\begin{tabular}{lcc}
\hline \hline & Case without channel & Case with channel \\
\hline$E_{p}(\mathrm{GeV})$ & 1.78 & 2.50 \\
$\Delta E / E(\%)$ & $100 \%$ & $28.5 \%$ \\
$\langle\theta\rangle($ degree $)$ & 5.46 & 5.13 \\
$\varepsilon(\mathrm{mm} \mathrm{mrad})$ & 3.08 & 2.77 \\
$N\left(10^{10} / \mu \mathrm{m}\right)$ & 7.36 & 9.18 \\
$E_{\text {tot }}\left(10^{10} \mathrm{GeV} / \mu \mathrm{m}\right)$ & 15.64 & 19.73 \\
\hline \hline
\end{tabular}

Meanwhile, the energy conversion efficiency is also improved by $26.2 \%$ for the high-energy protons $(\geq 1.5 \mathrm{GeV})$.

The scheme we proposed is robust as seen in Fig. 8, through the use of a plasma channel, and the quasimonoenergetic proton beams can be generated in all cases at $t=300 \mathrm{fs}$. We show the simulation results for different channel locations in Figs. 8(a) and 8(b), and it is found that as the distance $d$ increase from 0 to $16 \lambda$, the peak energy drops from 1.95 to $1.69 \mathrm{GeV}$, while the number of protons
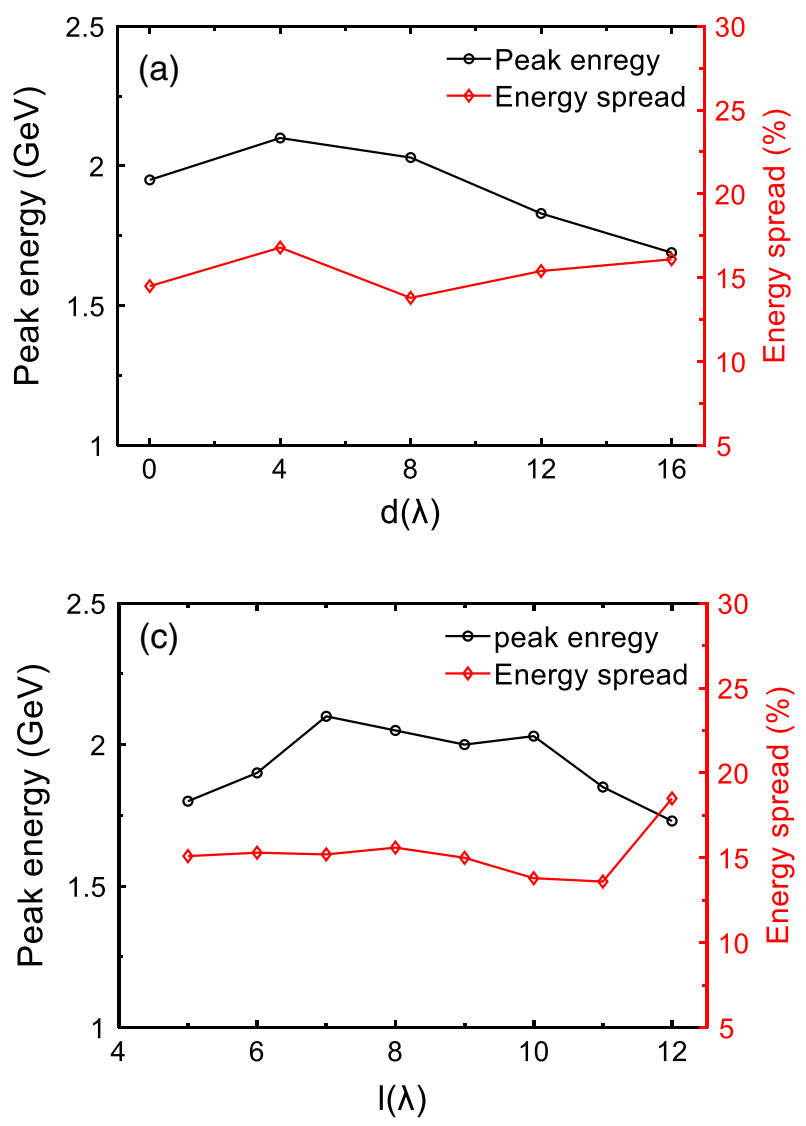

rises. We find that in the 2D geometry when $d$ is close to $8 \lambda$, the beam quality is better. Similarly, we also check the dependence on the channel width $l$, as presented in Figs. 8(c) and 8(d). We see that when the width $l$ is between $7 \lambda$ and $10 \lambda$, the peak energy is always larger than $2.0 \mathrm{GeV}$ and the corresponding energy spread is also lower, meaning there is little effect on beam quality. But for a much smaller width $(l=5 \lambda)$, the number of accelerated protons is declined. If a wider plasma channel $(l=12 \lambda)$ is employed, the peak energy falls from 2.0 to $1.73 \mathrm{GeV}$, while the number of protons remains nearly constant. For the present simulations, the width we suggested is $l \in[7 \lambda, 10 \lambda]$. All these simulations have shown the proton beams with high energy $(\sim 2.0 \mathrm{GeV})$, well-defined peak (energy spread $\leq 20 \%)$, and considerable particle number $(\sim 10 \mathrm{nC} / \mu \mathrm{m})$ can be produced by using an additional channel. Therefore, this scheme we proposed can effectively improve the beam quality of accelerated protons.

\section{SUMMARY}

In summary, in order to generate a high quality proton beam by weakening the mismatching effect in the later stage of RPA, a new scheme with an additional plasma
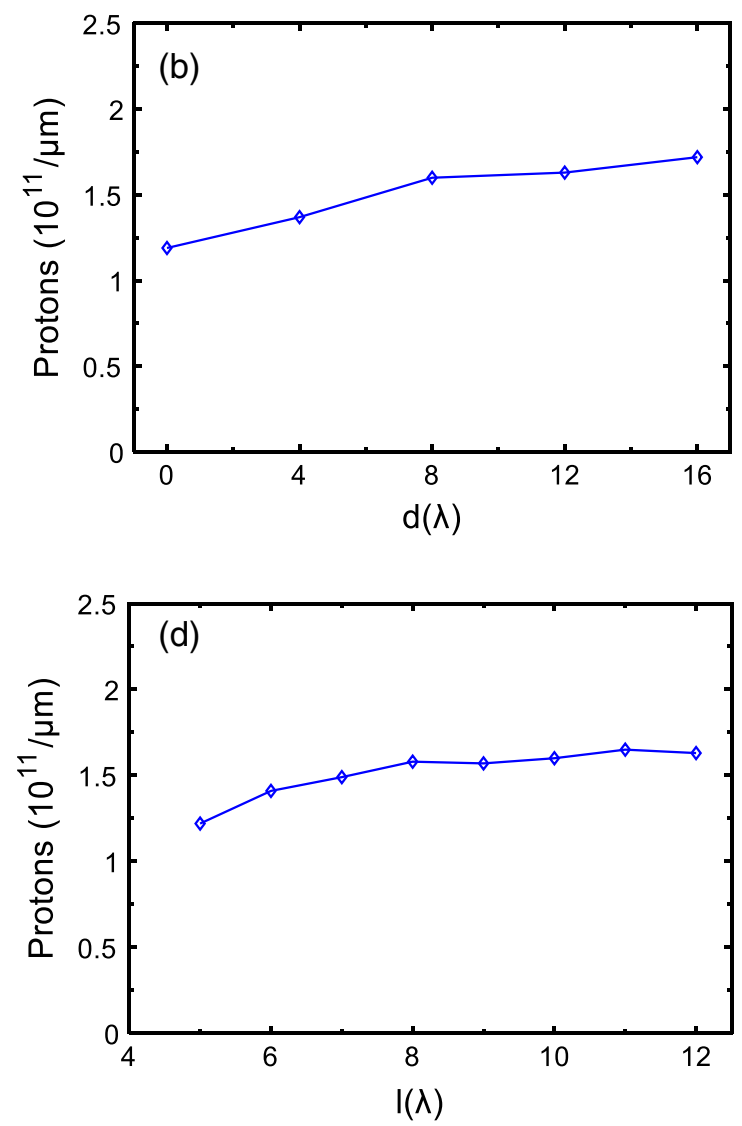

FIG. 8. Scaling of the peak energy, energy spread, and the number of protons against the plasma channel locations ( $d$ ) and wall widths ( $l$ ) at $t=300 \mathrm{fs}$. The simulations for scan are performed with the other parameters unchanged. Here, we choose the protons in the region of $-6 \lambda \leq y \leq 6 \lambda$. 
channel located behind a thin hydrogen foil has been proposed. The key point of this scheme is realizing a good local matching between the laser intensity profile and particle spatial distribution during the later stage, ensuring that protons can be stably accelerated by light pressure for a longer time. 2D PIC simulations show that the presence of a plasma channel can efficiently accelerate protons, and the peak energy and energy conversion efficiency for highenergy protons are improved by $40 \%$ and $26.2 \%$ compared to that in the case without a channel. In addition, the energy spread drops from $100 \%$ to $28.5 \%$. Therefore, this new scheme we suggested provides a more promising way to produce the high quality multi-GeV proton beams, which will favor further applications, such as nuclear physics and tumor therapy.

\section{ACKNOWLEDGMENTS}

This work is supported by National Natural Science Foundation of China (Grant No. 11775056).

[1] M. Roth, T. E. Cowan, M. H. Key, S.P. Hatchett, C. Brown, W. Fountain, J. Johnson, D. M. Pennington, R. A. Snavely, S. C. Wilks, K. Yasuike, H. Ruhl, F. Pegoraro, S. V. Bulanov, E. M. Campbell, M. D. Perry, and H. Powell, Fast Ignition by Intense Laser-Accelerated Proton Beams, Phys. Rev. Lett. 86, 436 (2001).

[2] A. Macchi, A. Antonicci, S. Atzeni, D. Batani, F. Califano, F. Cornolti, J. J. Honrubia, T. V. Lisseikina, F. Pegoraro, and M. Temporal, Fundamental issues in fast ignition physics: from relativistic electron generation to proton driven ignition, Nucl. Fusion 43, 362 (2003).

[3] M. Ghoranneviss, B. Malekynia, H. Hora, G. H. Miley, and $\mathrm{X}$. He, Inhibition factor reduces fast ignition threshold for laser fusion using nonlinear force driven block acceleration, Laser Part. Beams 26, 105 (2008).

[4] S. V. Bulanov, T. Zh. Esirkepov, V. S. Khoroshkov, A. V. Kuznetsov, and F. Pegoraro, Oncological hadrontherapy with laser ion accelerators, Phys. Lett. A 299, 240 (2002).

[5] F. Terranova, S. V. Bulanov, J. L. Collier, H. Kiriyama, and F. Pegoraro, Enabling pulse compression and proton acceleration in a modular ICF driver for nuclear and particle physics applications, Nucl. Instrum. Methods Phys. Res., Sect. A 558, 430 (2006).

[6] M. Borghesi, D. H. Campbell, A. Schiavi, M. G. Haines, O. Willi, A. J. MacKinnon, P. Patel, L. A. Gizzi, M. Galimberti, R. J. Clarke, F. Pegoraro, H. Ruhl, and S. Bulanov, Electric field detection in laser-plasma interaction experiments via the proton imaging technique, Phys. Plasmas 9, 2214 (2002).

[7] S.C. Wilks, A. B. Langdon, T.E. Cowan, M. Roth, M. Singh, S. Hatchett, M. H. Key, D. Pennington, A. MacKinnon, and R. A. Snavely, Energetic proton generation in ultra-intense laser-solid interactions, Phys. Plasmas 8, 542 (2001).

[8] H. Schwoerer, S. Pfotenhauer, O. Jäckel, K. U. Amthor, B. Liesfeld, W. Ziegler, R. Sauerbrey, K. W. D. Ledingham, and T. Esirkepov, Laser-plasma acceleration of quasimonoenergetic protons from microstructured targets, Nature (London) 439, 445 (2006).

[9] F. Wagner, O. Deppert, C. Brabetz, P. Fiala, A. Kleinschmidt, P. Poth, V. A. Schanz, A. Tebartz, B. Zielbauer, M. Roth, T. Stöhlker, and V. Bagnoud, Maximum Proton Energy above $85 \mathrm{MeV}$ from the Relativistic Interaction of Laser Pulses with Micrometer Thick $\mathrm{CH}_{2}$ Targets, Phys. Rev. Lett. 116, 205002 (2016).

[10] T. Esirkepov, M. Borghesi, S. V. Bulanov, G. Mourou, and T. Tajima, Highly Efficient Relativistic-Ion Generation in the Laser-Piston Regime, Phys. Rev. Lett. 92, 175003 (2004).

[11] X. Q. Yan, C. Lin, Z. M. Sheng, Z. Y. Guo, B. C. Liu, Y. R. Lu, J.X. Fang, and J. E. Chen, Generating High-Current Monoenergetic Proton Beams by a Circularly Polarized Laser Pulse in the Phase-Stable Acceleration Regime, Phys. Rev. Lett. 100, 135003 (2008).

[12] A. P. L. Robinson, M. Zepf, S. Kar, R. G. Evans, and C. Bellei, Radiation pressure acceleration of thin foils with circularly polarized laser pulses, New J. Phys. 10, 013021 (2008).

[13] N. Naumova, T. Schlegel, V. T. Tikhonchuk, C. Labaune, I. V. Sokolov, and G. Mourou, Hole Boring in a DT Pellet and Fast-Ion Ignition with Ultraintense Laser Pulses, Phys. Rev. Lett. 102, 025002 (2009).

[14] B. Qiao, M. Zepf, M. Borghesi, and M. Geissler, Stable GeV Ion-Beam Acceleration from Thin Foils by Circularly Polarized Laser Pulses, Phys. Rev. Lett. 102, 145002 (2009).

[15] S. V. Bulanov, E. Yu. Echkina, T. Zh. Esirkepov, I. N. Inovenkov, M. Kando, F. Pegoraro, and G. Korn, Unlimited Ion Acceleration by Radiation Pressure, Phys. Rev. Lett. 104, 135003 (2010).

[16] T. P. Yu, A. Pukhov, G. Shvets, and M. Chen, Stable LaserDriven Proton Beam Acceleration from a Two-Ion-Species Ultrathin Foil, Phys. Rev. Lett. 105, 065002 (2010).

[17] B. Qiao, M. Zepf, M. Borghesi, B. Dromey, M. Geissler, A. Karmakar, and P. Gibbon, Radiation-Pressure Acceleration of Ion Beams from Nanofoil Targets: The Leaky Light-Sail Regime, Phys. Rev. Lett. 105, 155002 (2010).

[18] L. Yin, B. J. Albright, B. M. Hegelich, K. J. Bowers, K. A. Flippo, T. J. T. Kwan, and J. C. Fernández, Monoenergetic and $\mathrm{GeV}$ ion acceleration from the laser breakout afterburner using ultrathin targets, Phys. Plasmas 14, 056706 (2007).

[19] L. Yin, B. J. Albright, D. Jung, R. C. Shah, S. Palaniyappan, K. J. Bowers, A. Henig, J. C. Ferńndez, and B. M. Hegelich, Break-out afterburner ion acceleration in the longer laser pulse length regime, Phys. Plasmas 18, 063103 (2011).

[20] L. Yin, B. J. Albright, K. J. Bowers, D. Jung, J. C. Fernández, and B. M. Hegelich, Three-Dimensional Dynamics of Breakout Afterburner Ion Acceleration Using High-Contrast Short-Pulse Laser and Nanoscale Targets, Phys. Rev. Lett. 107, 045003 (2011).

[21] E. L. Clark, K. Krushelnick, M. Zepf, F. N. Beg, M. Tatarakis, A. Machacek, M. I. K. Santala, I. Watts, P. A. Norreys, and A.E. Dangor, Energetic Heavy-Ion and Proton Generation from Ultraintense Laser-Plasma Interactions with Solids, Phys. Rev. Lett. 85, 1654 (2000). 
[22] E. Fourkal, I. Velchev, and C. M. Ma, Coulomb explosion effect and the maximum energy of protons accelerated by high-power lasers, Phys. Rev. E 71, 036412 (2005).

[23] H. Zhang, B. F. Shen, W. P. Wang, S. H. Zhai, S. S. Li, X. M. Lu, J. F. Li, R. J. Xu, X. L. Wang, X. Y. Liang, Y. X. Leng, R. X. Li, and Z.Z. Xu, Collisionless Shock Acceleration of High-Flux Quasimonoenergetic Proton Beams Driven by Circularly Polarized Laser Pulses, Phys. Rev. Lett. 119, 164801 (2017).

[24] A. Pak, S. Kerr, N. Lemos, A. Link, P. Patel, F. Albert, L. Divol, B. B. Pollock, D. Haberberger, D. Froula et al., Collisionless shock acceleration of narrow energy spread ion beams from mixed species plasmas using $1 \mu \mathrm{m}$ lasers, Phys. Rev. Accel. Beams 21, 103401 (2018).

[25] A. Higginson, R. J. Gray, M. King, R. J. Dance, S. D. R. Williamson, N. M. H. Butler, R. Wilson, R. Capdessus, C. Armstrong, J. S. Green et al., Near-100 MeV protons via a laser-driven transparency-enhanced hybrid acceleration scheme, Nat. Commun. 9, 724 (2018).

[26] W. J. Ma, I. J. Kim, J. Q. Yu, I. W. Choi, P. K. Singh, H. W. Lee, J. H. Sung, S. K. Lee, C. Lin, Q. Liao et al., Laser Acceleration of Highly Energetic Carbon Ions Using a Double-Layer Target Composed of Slightly Underdense Plasma and Ultrathin Foil, Phys. Rev. Lett. 122, 014803 (2019).

[27] T. P. Yu, Y. Y. Ma, M. Chen, F. Q. Shao, M. Y. Yu, Y. Q. $\mathrm{Gu}$, and Y. Yin, Quasimonoenergetic proton beam from ultraintense-laser irradiation of a target with holed backside, Phys. Plasmas 16, 033112 (2009).

[28] K. D. Xiao, C. T. Zhou, B. Qiao, and X. T. He, Guiding and collimation of laser-accelerated proton beams using thin foils followed with a hollow plasma channel, Phys. Plasmas 22, 093112 (2015).
[29] D. B. Zou, A. Pukhov, L. Q. Yi, H. B. Zhuo, T. P. Yu, Y. Yin, and F. Q. Shao, Laser-driven ion acceleration from plasma micro-channel targets, Sci. Rep. 7, 42666 (2017).

[30] C. Lv, F. Wan, Y. J. Hou, X. R. Hong, M. R. Jia, H. B. Sang, and B. S. Xie, Accelerating and guiding of $\mathrm{C}^{6+}$ by an intense laser irradiating on a foil target with a tapered channel, Phys. Plasmas 24, 083114 (2017).

[31] F. Pegoraro and S. V. Bulanov, Photon Bubbles and Ion Acceleration in a Plasma Dominated by the Radiation Pressure of an Electromagnetic Pulse, Phys. Rev. Lett. 99, 065002 (2007).

[32] M. Chen, A. Pukhov, T. P. Yu, and Z. M. Sheng, Enhanced Collimated GeV Monoenergetic Ion Acceleration from a Shaped Foil Target Irradiated by a Circularly Polarized Laser Pulse, Phys. Rev. Lett. 103, 024801 (2009).

[33] H. Y. Wang, X. Q. Yan, and M. Zepf, Collimated proton acceleration in light sail regime with a tailored pinhole target, Phys. Plasmas 21, 063113 (2014).

[34] M. Chen, A. Pukhov, Z. M. Sheng, and X. Q. Yan, Laser mode effects on the ion acceleration during circularly polarized laser pulse interaction with foil targets, Phys. Plasmas 15, 113103 (2008).

[35] T. D. Arber, K. Bennett, C.S. Brady, A. LawrenceDouglas, M. G. Ramsay, N. J. Sircombe, P. Gillies, R. G. Evans, H. Schmitz, A. R. Bell, and C. P. Ridgers, Contemporary particle-in-cell approach to laser-plasma modelling, Plasma Phys. Controlled Fusion 57, 113001 (2015).

[36] Y. J. Gu, Z. Zhu, X. F. Li, Q. Yu, S. Huang, F. Zhang, Q. Kong, and S. Kawata, Stable long range proton acceleration driven by intense laser pulse with underdense plasmas, Phys. Plasmas 21, 063104 (2014). 